\title{
Proporción sexual de la comunidad de pseudoescorpiones (Arachnida: Pseudoscorpiones) en bosque de manglar, San Antero- Córdoba, Caribe colombiano
}

\author{
Sex ratio of the community of pseudoscorpions (Arachnida: \\ Pseudoscorpiones) in mangrove forest, San Antero-Córdoba, Colombian \\ Caribbean
}

Bedoya-Roqueme, Edwin ${ }^{1 *}$ Biol, Quiros R, Jorge ${ }^{2}$ M.Sc.

${ }^{1}$ Universidad de Córdoba, Colombia. ${ }^{2}$ Universidad de Córdoba, Programa de Biología, Facultad de Ciencias Básicas, Colombia.

\section{Keywords:}

Microhabitat;

structure;

phenology;

cycle of life;

sexual dimorphism.

\section{Abstract}

In populations of pseudoscorpions in fragments of mangrove forest of the department of Córdoba, Colombian, Caribbean, was carried out a study on sex ratio and periods of reproduction. A total of 1,037 individuals were collected, distributed in five families and six species. Most of the species presented a sex ratio 1:1, and periods of reproduction univoltine, however, Epactiochernes sp., it registered a sex ratio slanted toward the females and Serianus aff. carolinensis, present periods bivoltines. The sex ratio exhibited by most of the species and the periods of reproduction, they suggest that its bound selective strategy to the reproduction, is adjusted to the climatic season of the mangrove forests, related with the exclusivity of the species in the microhabitat where they are unwrapped.

\section{Palabras Clave:}

Microhábitat: estructura; fenología; ciclo de vida; dimorfismo sexual.

INFORMACIÓN Recibido: 04-10-2015; Aceptado: $27-06-2016$. Correspondencia autor: roquemeedj@gmail.com

\section{Resumen}

En poblaciones de pseudoescorpiones en fragmentos de bosque de manglar del departamento de Córdoba, Caribe colombiano, se realizó un estudio sobre proporción sexual y periodos de reproducción. Se colectaron 1.037 individuos distribuidos en cinco familias y seis especies. La mayoría de las especies presentó una proporción sexual 1:1, y periodos de reproducción univoltinos, sin embargo, Epactiochernes sp., registró una proporción sexual sesgada hacia las hembras y Serianus aff. carolinensis, presento periodos bivoltinos. La proporción sexual exhibida por la mayoría de las especies y los periodos de reproducción sugieren que, su estrategia selectiva ligada a la reproducción, se encuentra ajustada a las épocas climáticas de los bosques de manglar, relacionado con la exclusividad de las especies en los microhábitat donde se desenvuelven. 


\section{Introducción}

La proporción sexual, se define como la razón entre el número de machos y hembras en una población, es aceptado, que en muchos ensamblajes de invertebrados se presente igual número de individuos en ambos sexos (OMOLOYE, 2006; TABADKANI et al., 2012). Los postulados de FISHER (1930) explican que la proporción sexual de la mayoría de las especies tiende a ser uno a uno, sin embargo, se ha observado que algunas especies se desvían de esta proporción, para aumentar al máximo el potencial evolutivo y las perspectivas a largo plazo de una población dada (HJERNQUIST et al., 2009; TABADKANI et al., 2012; WEDEKIND, 2012). En este sentido, diferencias en el comportamiento de los individuos, en el tamaño o la morfología, se consideran, factores que pueden estar sesgando la proporción sexual en aquellas especies que presentan determinación a nivel genético (BUNNEFELD et al., 2009; TRYJANOWSKI et al., 2009; WEDEKIND, 2012).

Los pseudoescorpiones, son arácnidos mesodiversos que habitan todos los ecosistemas terrestres, siendo diversos en los trópicos y subtrópicos (WEYGOLDT, 1969; JUDSON, 1994; DEL-CLARO y TIZOPEDROSO, 2009; HARVEY, 2013). Son vivíparos y presentan variados grados de cuidados para sus crías (WEYGOLDT, 1969; DEL-CLARO y TIZO-PEDROSO, 2009). Varios géneros, se han reportado exclusivos de ambientes marinos y de la vegetación de costa (HOFF, 1959; GABBUTT, 1962; WEYGOLDT, 1969; MAHNERT y SCHUSTER, 1981; LEE, 1979; HARVEY, 2009; MAHNERT, 2014). Sin embargo, los datos ecológicos son muy escasos, a pesar de investigaciones realizadas en zonas litorales (GABBUTT, 1962; 1970; LEE, 1979; MAHNERT y SCHUSTER, 1981; HARVEY, 2009).

Entre los arácnidos, las arañas de la familia Araneidae y Theridiidae exhiben una proporción sexual sesgada hacia las hembras, mientras que Pholcidae presenta una proporción 1:1; la mayoría de Opiliones presentan ciclos de vida anuales comportamiento similar se ha observado en los escorpiones que exhiben igual proporción de hembras y machos al nacimiento y extendida entre todos los arácnidos (FOELIX, 2011; BELOZEROV, 2013). A pesar de lo anterior, poco se conoce acerca de la proporción sexual de estos arácnidos en ambientes marinos, y solo se conocen datos sobre fenología de varias regiones de Europa y en bosques de la Amazonia (SCHUSTER, 1962; GABBUTT, 1966; GABBUTT,1970; KENSLER, 1967; WEYGOLDT, 1969; ADIS y MAHNERT, 1985; ADIS et al., 1988; ADIS y MAHNERT, 1993; MORÁIS y ADIS, 1997; ADIS y JUNK, 2002; RANIUS y DOUWES, 2002; VANIN y TURCHETTO, 2007; BELOZEROV, 2013).
Artrópodos que habitan bosques inundables, han desarrollados estrategias para compensar la pérdida periódica de su hábitat terrestre, asimismo, algunas poblaciones pueden haber sufrido cambios por el aislamiento de los bosques y los cambios en las épocas climáticas; como se ha indicado para los pseudoescorpiones, que presentan ciclos de vida muy particulares, influenciados por diferentes factores que generan estacionalidad en las etapas de desarrollo post-embrionarias y solapamiento de generaciones e influyendo indirectamente en la proporción sexual de las poblaciones, debido que, algunas especies exhiben proporciones sexuales, ajustadas a las épocas climáticas (GABBUTT; 1970; ADIS et al., 1988; ZEH 1987a; 1987b ; ZEH y ZEH, 1990; 1997; BELOZEROV, 2013).

Por tanto, se consideró fundamental conocer y establecer, la estructura, el dimorfismo sexual y la proporción sexual de estos arácnidos en ambientes marinos; en este sentido, la proporción sexual de la comunidad de pseudoescorpiones, el dimorfismo sexual y los periodos de reproducción en los fragmentos de bosque de manglar del departamento de Córdoba, Caribe Colombiano, son presentados en el siguiente estudio.

\section{Materiales y métodos}

Área de estudio. El municipio de San Antero, se encuentra ubicado en el costado suroeste del golfo de Morrosquillo, entre los $9.7^{\circ} 24^{\prime} 34.29^{\prime \prime} \mathrm{N}$ y $75^{\circ} 45^{\prime} 20.95^{\prime \prime}$ $\mathrm{W}$; con una temperatura promedio de $28^{\circ} \mathrm{C}$, una precipitación promedio de $1.337 \mathrm{~mm}$ anuales y un ciclo unimodal-biestacional dominante en la zona (CVS-INVEMAR, 2010; CORTÉS y RANGEL, 2011). Se escogieron cinco fragmentos, ubicados en la línea de costa. La selección de los sitios, obedece a características relacionadas con su estado de conservación, la estructura vegetal y fácil acceso (Fig. 1).

Colecta de los individuos. Para la colecta de los pseudoescorpiones, se adaptaron y fueron aplicadas las metodologías descritas por GABBUTT (1970), MAHNERT y ADIS (2002) y las recomendaciones realizadas por ADIS et al. (1988). Entre marzo y octubre de 2014 , se realizó el muestreo abarcando los periodos climáticos. Como unidad de muestreo se utilizó un cuadrante de $1 \mathrm{~m}^{2}$, ubicados perpendicularmente a la línea de costa y replicado tres veces por fragmento para un total de 15 unidades; la hojarasca colectada se depositó en bolsas selladas para evitar perdida de humedad (ROJAS, 1989). Los pseudoescorpiones fueron extraídos utilizando el procesamiento por embudo de Berlese. A través de la colecta manual, 
se examinó la corteza de 20 troncos por sector, para un total de 800 troncos examinados, por un tiempo efectivo de $60 \mathrm{~min} . /$ colector, los especímenes fueron depositados en frascos que contenían alcohol al $70 \%$ como preservante para su posterior revisión en laboratorio.

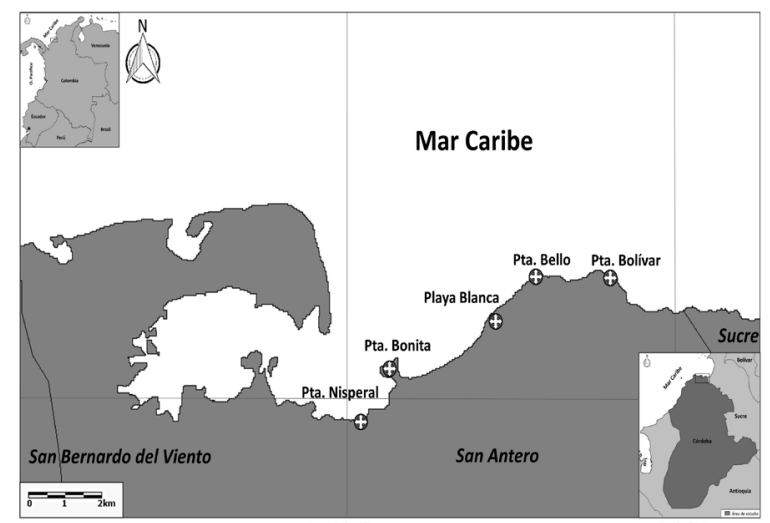

Figura 1. Área de estudio y localización de los fragmentos de bosque de manglar en la línea de costa del municipio de San Antero.

Fase de laboratorio. Previamente a someter los especímenes a un proceso de aclaramiento por difusión lenta utilizando ácido láctico $70 \%$, se diseccionaron una pata I y otra IV, un quelícero y un pedipalpo, de éste se le ha separado la pinza (JUDSON, 1992). Los artejos sueltos se han guardado en un microvial y conservados en alcohol $70 \%$ en el mismo vial que el resto de cada ejemplar. Para la identificación, se han empleado montajes no permanentes en glicerina, proceso realizado en el Laboratorio de Entomología de la Universidad de Córdoba, con la ayuda de un microscopio Carl Zeiss, plus Axiostar, Alemania. Posteriormente, la medición de los especímenes se realizó en las instalaciones del laboratorio de microscopia de la Universidad de Córdoba, utilizando un micrómetro incorporado al microscopio (Carl Zeiss, Axiostar, Alemania) y con la ayuda del programa Axio visión 4.8.2. SP3 (CARL ZEISS, 2013).

El material hace parte de la colección del Instituto de Ciencias Naturales-ICN de la Universidad Nacional de Colombia-sede Bogotá con código ICN-APs-391 e ICNAPs-392 y la colección del laboratorio de Entomología de la Universidad de Córdoba con códigos desde Ps001 a Ps-006.

Proporción sexual. Los pseudoescorpiones colectados en cada sector de estudio, durante toda la investigación, se clasificaron como ninfas (protoninfa, deutoninfa y tritoninfa) o adulto (ADIS et al., 1988), teniendo en cuenta la nomenclatura de los tricobotrios propuesta por CHAMBERLIN (1931) para cada estadio y las categorías de hembra y macho teniendo en cuenta la forma de la placa genital, de acuerdo con LEGG (1974; 1975) y las claves fotográficas de BUDDLE (2010).

Análisis de datos. La proporción sexual fue calculada utilizando la ecuación [H/H+M] (SMITH, 1999). Para el análisis de la proporción sexual, para cada una de las especies, se calculó Chi-cuadrado $\left(\mathrm{X}^{2}\right)$ con sus respectivas tablas de contingencia, para observar si presentaban diferencias entre el número de machos y hembras. Adicional a esto, se utilizaron las pruebas t-Student y W de man-Withney, para comparar la longitud corporal y evidenciar, dimorfismo sexual entre machos y hembras.

\section{Resultados}

Se colectó un total de 1.037 individuos, distribuidos en cinco familias y seis del orden Pseudoscorpiones, Olpiidae fue la familia más abundante con 775 individuos, seguida de la familia Lechytiidae con 188 individuos y Garypinidae con 66 individuos, mientras que las familias menos abundantes fueron Chthoniidae y Chernetidae con seis y cinco individuos respectivamente.

Proporción sexual de la comunidad de pseudoescorpiones. Se colectaron 583 adultos, de los cuales, se categorizaron 294 machos y 289 hembras (Tabla 1). De igual forma, se colectaron 454 ninfas, de los cuales, 181 fueron protoninfas (39,8\%), 109 deutoninfas $(24 \%)$ y 164 tritoninfas $(36,1 \%)$ (Tabla 2). El fragmento de bosque de manglar de Playa Blanca, registró el mayor porcentaje de ninfas (50\%), seguido del fragmento de Punta Bolívar (24\%), Punta Bello (13,3\%) y Punta Bonita (12,3\%); en contraste, el fragmento de Punta Nisperal registró el menor porcentaje de ninfas con $(0,2 \%)$.

Pachyolpium isolatum Beier (1931). registró el mayor número de machos 173 y de hembras 131 , en promedio la longitud corporal de las hembras fue de $(2,69 \pm 0,29$ $\mathrm{mm})$ y los machos $(2,08 \pm 0,18 \mathrm{~mm}$ ) (Tabla 2$)$. Se presentaron diferencias en la longitud corporal $(\mathrm{W}=$ $21269,0 ; \alpha=0,05 ; P<0,05)$ evidenciando dimorfismo sexual entre los individuos (Tabla 2). La proporción de sexos observada durante todo el estudio, fue de 1:1, no se presentaron diferencias significativas entre el número de machos y hembras $\left(X^{2}=12,3 ; G I=7 ; P=0,093\right)$; $\sin$ embargo, se registraron fluctuaciones entre el número de individuos durante los meses de estudio, a pesar de esto, la proporción entre sexos se mantuvo (Figura 2A). 
Tabla 1. Estructura-edad de los pseudoescorpiones colectados.

\begin{tabular}{|c|c|c|c|c|c|c|}
\hline \multirow[t]{2}{*}{ Especies } & \multicolumn{2}{|c|}{ Protoninfas } & \multicolumn{2}{|c|}{ Deutoninfas } & \multicolumn{2}{|c|}{ Tritoninfas } \\
\hline & Abundancia & Promedio & Abundancia & Promedio & Abundancia & Promedio \\
\hline Epactiochernes sp. & 0 & 0 & 0 & 0 & 1 & 0.12 \\
\hline Lechytia chthoniiformis & 0 & 0 & 0 & 0 & 10 & 1.25 \\
\hline Pachyolpium isolatum & 142 & 17,7 & 96 & 12 & 139 & 17.3 \\
\hline Paraliochthonius quirosi & 0 & 0 & 0 & 0 & 0 & 0 \\
\hline Planctolpium arboreum & 2 & 0.25 & 8 & 1 & 6 & 0.75 \\
\hline Serianus aff. carolinensis & 0 & 0 & 1 & 0.12 & 1 & 0.12 \\
\hline
\end{tabular}

Tabla 2. Longitud corporal (medidas en $\mathrm{mm}$ ) de las especies de pseudoescorpion, indicando sexo y especie

\begin{tabular}{|c|c|c|c|c|c|}
\hline Sexos & Abundancia & Promedio & Desviación Estándar & Mínimo & Máximo \\
\hline \multicolumn{6}{|c|}{ Pachyolpium isolatum (Olpiidae) } \\
\hline Machos & 173 & 2,08 & 0,19 & 1,7 & 2,9 \\
\hline Hembras & 131 & 2,69 & 0,29 & 1,9 & 3,1 \\
\hline \multicolumn{6}{|c|}{ Lechytia chthoniiformis (Lechytiidae) } \\
\hline Machos & 77 & 0,85 & 0,06 & 0,7 & 1 \\
\hline Hembras & 67 & 1,03 & 0,11 & 0,7 & 1,3 \\
\hline \multicolumn{6}{|c|}{ Serianus aff. carolinensis (Garypinidae) } \\
\hline Machos & 23 & 1,7 & 0,24 & 1,3 & 2,1 \\
\hline Hembras & 20 & 2,3 & 0,36 & 1,9 & 2,9 \\
\hline \multicolumn{6}{|c|}{ Planctolpium arboreum (Olpiidae) } \\
\hline Machos & 19 & 1,6 & 0,25 & 1,2 & 2,0 \\
\hline Hembras & 16 & 1,9 & 0,26 & 1,5 & 2,5 \\
\hline \multicolumn{6}{|c|}{ Paraliochthonius quirosi (Chthoniidae) } \\
\hline Machos & 2 & 1,1 & 0,14 & 1,0 & 1,2 \\
\hline Hembras & 4 & 1,25 & 0,1 & 1,1 & 1,3 \\
\hline
\end{tabular}

Lechytia chthoniiformis Balzan (1890): se categorizaron 67 hembras y 77 machos; en promedio, las hembras midieron $(1,0 \pm 0,11 \mathrm{~mm})$ y los machos $(0,85 \pm 0,06 \mathrm{~mm})$ y presentaron diferencias significativas en la longitud corporal ( $W=4690,5 ; \alpha=0,05 ; P<0,05)$, evidenciando dimorfismo sexual (Tabla 2). La proporción de sexos observada durante todo el estudio, fue de 1:1, no se presentaron diferencias significativas entre el número de machos y hembras $\left(X^{2}=12,5 ; \mathrm{Gl}=6 ; \mathrm{P}=0,93\right)$ (Figura 2B).

Serianus aff. carolinensis Muchmore (1968): registró 23 hembras y 20 machos, las hembras midieron en promedio $(2,35 \pm 0,36 \mathrm{~mm})$ y los machos $(1,77 \pm 0,24$ $\mathrm{mm})$, se presentó diferencias en la longitud corporal $(W=426,0 ; \alpha=0,05 ; P<0,001)$, lo cual, evidencia un verdadero dimorfismo sexual (Tabla 2). La proporción de sexos observada durante todo el estudio, fue de 1:1, no se presentaron diferencias entre el número de machos $y$ hembras $\left(X^{2}=14.1 ; G I=7 ; P=0.57\right)$. Esta especie, a diferencia de las otras, exhibe un comportamiento particular (Fig. 2C).

Planctolpium arboreum Hoff (1964): registró 19 machos y 16 hembras, las hembras midieron en promedio $(1,92 \pm 0,26 \mathrm{~mm})$ y los machos $(1,56 \pm 0,25$ $\mathrm{mm})$, presentando diferencias en la longitud corporal $(t=-4,08 ; P<0,01)$, evidenciando dimorfismo sexual significativo con base en las medidas corporales (Tabla 2). La proporción de sexos observada durante todo el estudio, fue de 1:1 (Fig. 2D), no se presentaron diferencias entre el número de machos y hembras $\left(X^{2}=9,48 ; G \mathrm{G}=4 ; \mathrm{P}=0,765\right)$.

Paraliochthonius quirosi Bedoya (2015): registró solo dos machos y cuatro hembras, siendo una de las especies que presentó el menor número de hembras y machos, la longitud corporal de las hembras midió en promedio $(1,25 \pm 0,1 \mathrm{~mm})$ y los machos $(1,1 \pm 0,14)$, sin presentarse diferencias en la longitud corporal $(\mathrm{W}=$ $7.0 ; \alpha=0.05 ; P=0.22$ ) (Tabla 2). La proporción de sexos observada durante todo el estudio, fue de 1:1, no se presentaron diferencias en el número de machos $y$ hembras $\left(X^{2}=3,841 ; \mathrm{Gl}=1 ; \mathrm{P}=0,43\right)$ (Fig. $\left.2 \mathrm{E}\right)$.

Epactiochernes sp: presentó una proporción de sexos observada durante todo el estudio de 3:1 (Fig. 2F), a pesar de lo anterior, no se presentaron diferencias en el número de machos y hembras $\left(X^{2}=7.815, G l=3\right.$, $\mathrm{P}=0.261)$. La especie Epactiochernes sp., registró solo un macho y tres hembras, la longitud corporal de las 

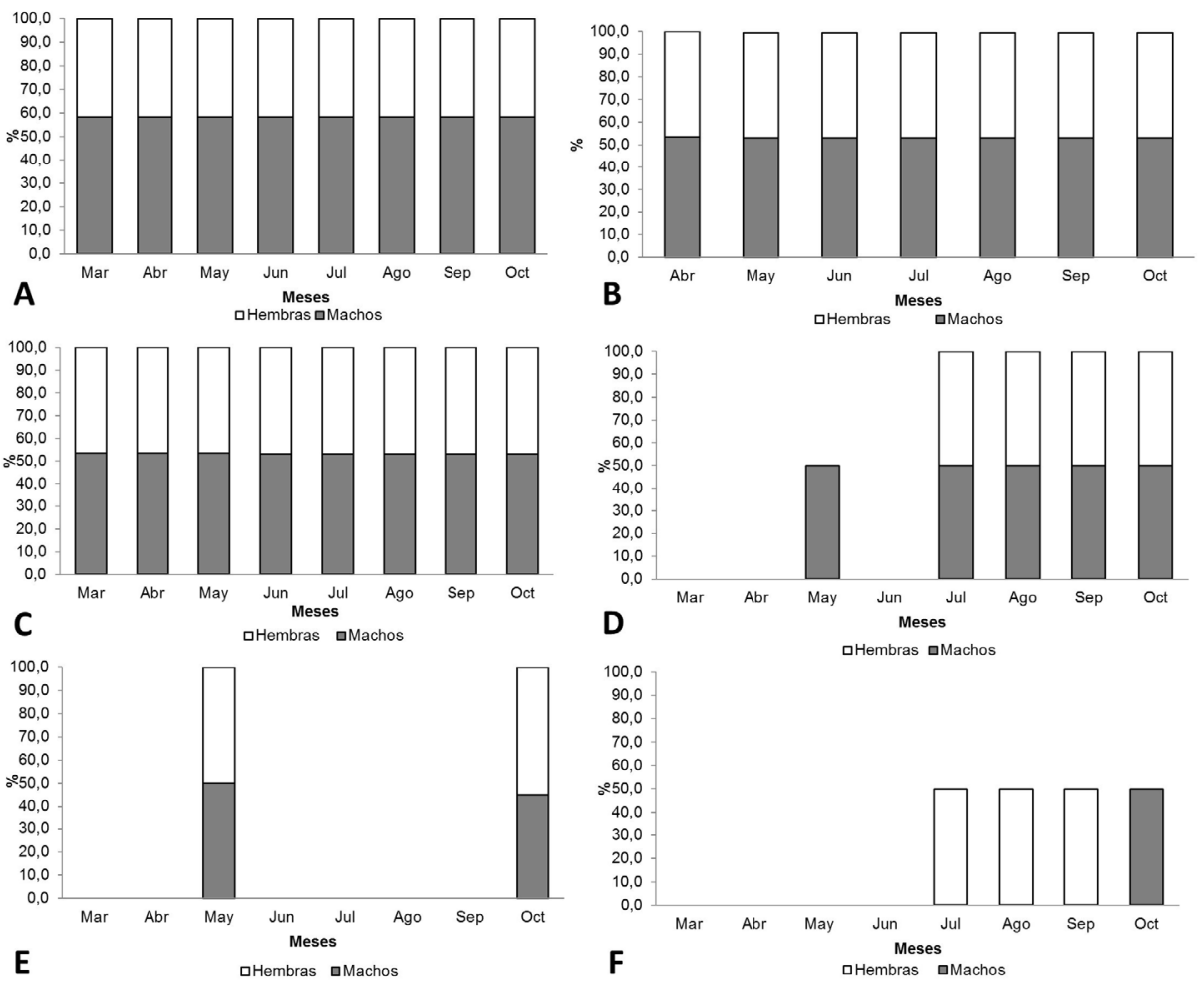

Figura 2. Proporción sexual de las especies de pseudoescorpiones: A, Pachyolpium isolatum. B, Lechytia chthoniiformis. C, Serianus aff. carolinensis. D, Planctolpium arboreum. E, Paraliochthonius quirosi. $\mathbf{F}$, Epactiochernes sp.

hembras midió en promedio $(1,9 \pm 0,35 \mathrm{~mm})$ y el macho $1,5 \mathrm{~mm}$, no se presentaron diferencias en la longitud corporal ( $W=9.0 ; \alpha=0.05 ; P=0.06)$; sin embargo, debido al bajo número de individuos registrados, no se puede descartar que se presente un dimorfismo sexual marcado.

Periodos de reproducción: Se comparó el número de hembras y machos (adultos) con el número de ninfas, encontrándose una variación muy marcada para todas las especies como sigue:

En la especie $P$. isolatum, el mayor número de ninfas se registró desde marzo hasta junio con un promedio de 67 $\pm 3,6$ ind./mes, en contraste, en estos meses el número de adultos (hembras y machos) presentó los valores más bajos de abundancia, con un promedio de $35 \pm 12,9$ ind./mes; al parecer, estos meses pueden corresponder con el último periodo de desarrollo postembrionario. El número de ninfas, tuvo un descenso muy marcado en el mes de junio y un incremento considerable de los individuos adultos, desde agosto hasta octubre con un promedio de $47 \pm 13,2$ ind./mes; lo cual, permite asegurar que durante estos meses se puede presentar el pico de reproducción de la especie, siendo septiembre el mes con el mayor número de adultos, exhibiendo un comportamiento univoltino (Figura 3A).
La especie L. chthoniiformis, durante todo el estudio registró mayor número de machos y hembras (adultos) que el presentado por las ninfas, con un promedio de $35 \pm 12,3$ ind./mes (Figura 2B). Entre abril y junio, no se registraron ninfas, meses en los que se presentaron los menores valores de abundancia para los adultos, lo cual sugiere, que la tendencia es encontrar más adultos que ninfas. Asimismo, los mayores valores de abundancia se registraron entre agosto y octubre, con un promedio de $30 \pm 3,1$ ind./mes, considerando estos meses como el pico de reproducción de esta especie, lo cual indica, que presenta un comportamiento univoltino (Figura 3B).

Se registraron dos periodos de máxima abundancia para hembras y machos (adultos) para la especie Serianus aff. carolinensis (Figura 3C). Entre marzo y mayo no se registraron ninfas, la primera deutoninfa se colectó en junio y en el mes de julio se colectó una tritoninfa. El primer pico de reproducción, fue identificado entre abril y mayo con un promedio de $8 \pm 1$ ind./mes, cabe resaltar, que en estos meses hasta junio se registraron hembras con sacos ovígeros (Figura $3 C)$. En el mes de julio se registró un descenso en el número de ninfas; sin embargo, entre julio y agosto se incrementó nuevamente los valores de abundancia de los adultos, con un promedio de $4 \pm 1,2$ ind./mes (Figura 

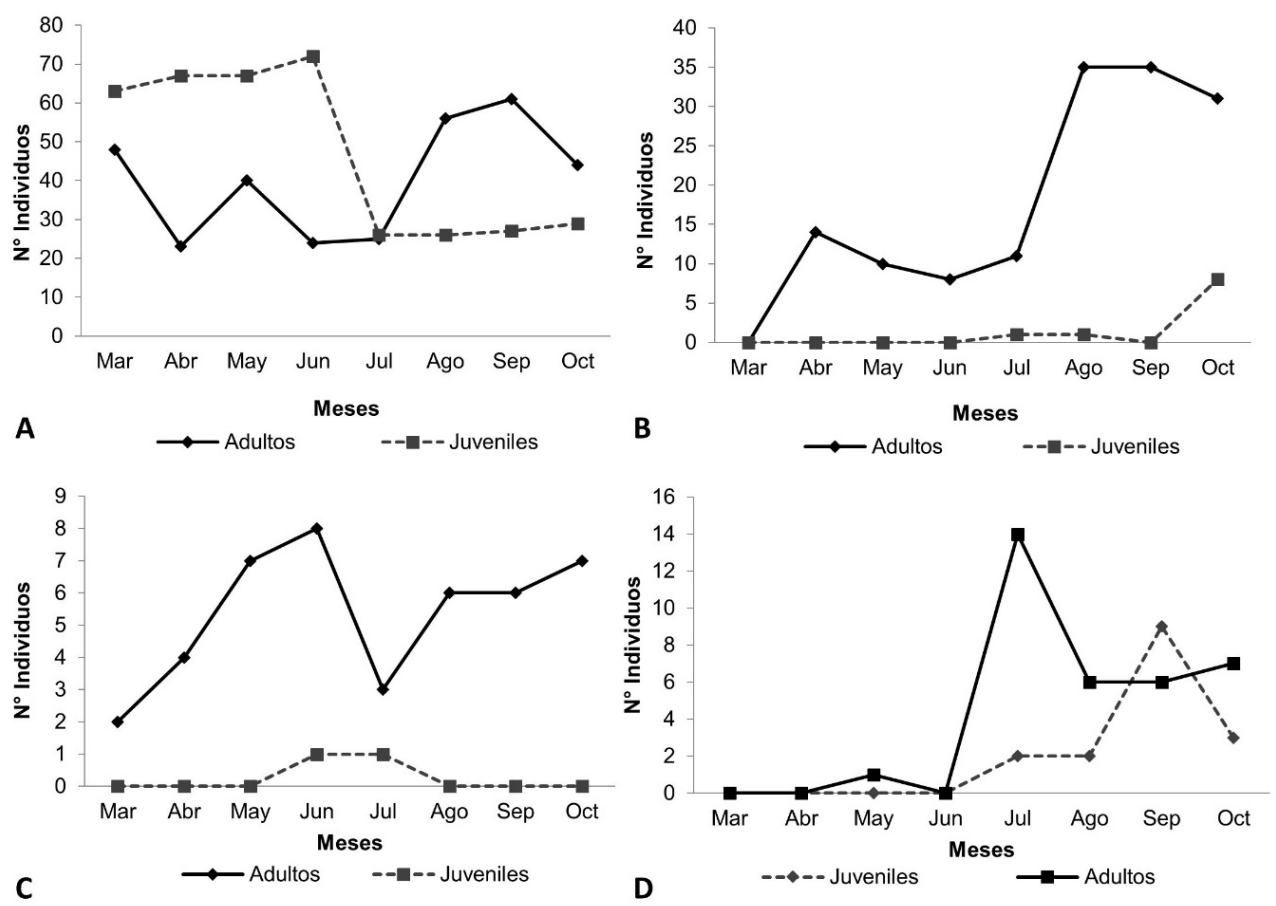

Figura 3. Fluctuación del número de adultos (hembras y machos) y de ninfas: A, Pachyolpium isolatum. B, Lechytia chthoniiformis. C, Serianus aff. carolinensis. D, Planctolpium arboretum

3C). En el mes de octubre aumentó considerablemente la abundancia, con un promedio de $6 \pm 0,9$ ind./ mes, donde se volvió a registrar hembras con sacos ovígeros, siendo septiembre y octubre el segundo pico de reproducción; este comportamiento, demuestra que Serianus aff. carolinensis es bivoltino (Figura 3C).

La especie $P$. arboreum, en el mes de mayo se presentó el valor más bajo de abundancia y para el mes de junio solo se registraron ninfas (Figura 3D). La tendencia, sugiere que se puede encontrar igual número de machos y de hembras. El mes de Julio registró el mayor número de machos y hembras (adultos), con un promedio de $7 \pm 4,9$ ind./mes (Figura 3D). En este periodo, se registraron hembras ovadas y en cámaras de seda y coincidió con el registro de ninfas y un aumento en su abundancia, con un promedio de $4 \pm$ 2,8 ind./mes; este comportamiento, sugiere que esta especie es univoltina (Figura 3D).

Se registró poca actividad para la especie $P$. quirosi, debido a esto, los valores más altos de machos y hembras se registraron en mayo y octubre, no se registraron ninfas y hembras con sacos ovígeros, por tanto, se hizo difícil el registro de los respectivos instar, así como, identificar su periodo de reproducción. A pesar de que se registraron pocos individuos y la particular actividad de Epactiochernes sp., la tendencia, muestra que se pueden encontrar mayor número de hembras que de machos, siendo octubre el mes donde se registró el único macho exhibiendo un comportamiento críptico, debido a esto, no se puede asegurar con certeza, el periodo de reproducción de esta especie.

\section{Discusión}

En los cinco fragmentos de bosque de manglar evaluados, la mayoría de las especies registradas exhibieron una proporción sexual 1:1; autores como GABBUTT (1970) y POLIS (1990), consideran que esta proporción se encuentra bien extendida entre los arácnidos. Sin embargo, se han reportado marcadas variaciones entre las arañas, Anelosimus eximius Keyserling, 1884 (Theridiidae) presenta una proporción sexual que favorece a las hembras de 5:1; mientras que Larinioides cornutus (Clerk, 1757) (Araneidae) y especies de la familia Pholcidae (Carapoia ocaina Huber, 2000 y Mesabolivar aurantiacus Mello-Leitao, 1930) exhiben una proporción 1:1; mientras que, Metagonia taruma Huber, 2000 presenta diferencias que se atribuyen a variaciones estocásticas (VOLLRATH, 1986; YSNEL, 1991; MACHADO et al., 2007; CARVALHO et al., 2010; FOELIX, 2011). De igual forma, algunas especies de escorpiones presentan una proporción sexual que favorece a las hembras de 1:3 y de opiliones Megabunus diadema (Fabricius, 1799) (Phalangiidae) se considera partenogénica, sin embargo, especies de Prionostemma (Opilionida) presentan una proporción 1:1 (MERINO-SÁINZ et al., 2013; GRETHER et al., 2014; 
KASSIRI et al., 2015). A pesar de esto, la proporción de sexos presente en la naturaleza, tiende a ser siempre 1:1 e indica roles de igual importancia entre machos y hembras, dado que, la selección podría minimizar al sexo menos productivo, por tanto, la proporción sexual 1:1 sugiere, que el costo de criar con éxito las ninfas, es más o menos el mismo independientemente de su sexo (FISHER, 1930; SMITH, 1999; WADE et al., 2003; TABADKANI et al., 2012; WEDEKIND, 2012).

En este sentido, Epactiochernes sp., fue la única especie que presentó una proporción sexual de $3: 1$, lo cual sugiere, por la tendencia mostrada, que presenta una proporción sesgada hacia las hembras; ahora bien, el bajo número machos registrados, se atribuye al habito críptico de especie, comportamiento observado en otros artrópodos, donde, los machos construyen sitios profundos de anidación en el sustrato, y concuerda con lo registrado para algunas especies de pseudoescorpiones, como Paratemnoides nidificator (Atemnidae) que exhibe una proporción sexual sesgada hacia las hembras de 2:3 y Allochernes dubius (Chernetidae) de 1:3 en época de invierno y de 1:9 en época de cría; de igual forma, hay excepciones en aquellas especies que presentan competencia por compañeros para generar descendencia y se ha observado, que la combinación de hábitat y la sesgada proporción sexual operacional, podría generar intensa competencia de los machos (HAMILTON 1967; GABBUTT, 1970; ZEH 1987a; 1987b; ZEH y ZEH, 1990; WADE et al., 2003; TIZO-PEDROSO y DEL-CLARO, 2007).

En muchas especies de pseudoescorpiones, los machos y las hembras son fácilmente distinguibles por su longitud corporal, siendo, los machos más pequeños que las hembras evidenciando un dimorfismo sexual y otros caracteres sexuales secundarios (WEYGOLDT, 1969; ZEH y ZEH, 1997). Sin embargo, especies como L. chthoniiformis (Lechytiidae) y P. quirosi (Chthoniidae), en los fragmentos de bosque de manglar evaluados no presentaron diferencias significativas en la longitud corporal y no evidencian un verdadero dimorfismo sexual, lo cual se atribuye al microhábitat que ocupan, L. chthoniiformis presente en el suelo del manglar y $P$. quirosi críptico, asociado al cascajo de coral y la mayor parte del día sumergido por agua del mar. Por tanto, en aquellas especies de pseudoescorpiones que no presentan competencias por las hembras, la selección actúa favoreciendo el rápido desarrollo de la atapa adulta y el tamaño corporal para tener acceso a las hembras (WEYGOLDT, 1969; ZEH y ZEH, 1990; 1992; 1997).

De igual forma, $P$. isolatum, $P$. arboreum, Serianus aff. carolinensis, en los fragmentos de bosque de manglar evaluados, exhiben un verdadero dimorfismo sexual basado en las diferencias significativas de la longitud corporal, atribuido a los microhábitat que ocupan, $P$. isolatum en la hojarasca, $P$. arboreum en la corteza de troncos en descomposición y la especie Serianus aff. carolinensis se encuentra separada por tiempo y espacio (siendo exclusiva de corteza de árboles de zonas inundables), sin generar, competencia con otras especies de pseudoescorpiones por los recursos, exhiben una conducta intermedia, de acuerdo, a la utilización del hábitat permiten la deposición de miles de espermatóforos en compensación por el bajo éxito de fertilización de la hembra y se ve reflejado en alcanzar un esfuerzo reproductor temprano, como producto una rápida etapa adulta y un menor tamaño por parte de los machos (WEYGOLDT, 1969; ZEH y ZEH, 1990; 1992; 1997).

En general, las especies presentaron un comportamiento univoltino en los fragmentos de bosque de manglar con mayor periodo reproductivo, entre los meses de julio/ agosto y agosto/septiembre, tanto en la hojarasca como en el suelo, al comienzo de la época de lluvia, utilizando la corteza de los arboles como sitios de anidación y refugio para las ninfas. Algunos autores, sugieren, que los pseudoescorpiones ajustan su ciclo de vida, como su periodo de reproducción, de acuerdo a las épocas y/o estaciones climáticas (como GABBUTT y VACHON, 1965; WEYGOLDT, 1969; ADIS y MAHNERT, 1985; ADIS et al., 1988; ADIS y MAHNERT, 1990; ADIS y MAHNERT, 1993; MORÁIS y ADIS, 1997; ADIS y JUNK, 2002; AGUIAR y BÜHRNHEIM, 2003; AGUIAR et al., 2006; BELOZEROV, 2013).

Sin embargo, Serianus aff. carolinensis exhibió un comportamiento bivoltino con mayor periodo de reproductivo en la corteza de árboles de zonas inundables entre los meses de junio/julio durante la época de transición y un segundo periodo menor entre los meses de octubre/noviembre durante la época seca. Autores como ADIS et al. (1988), sugieren que algunas poblaciones de pseudoescorpiones que habitan áreas inundables, presentan estrategias que compensan sus periodos perdidos de hábitos terrestres, en este caso, Serianus aff. carolinensis se encuentra adaptado morfológica y fisiológicamente para habitar las zonas inundables del manglar y concuerda, con lo registrado en bosques de la Amazonia y de Europa donde se ha encontrado que las poblaciones de pseudoescorpiones se encentran adaptados para ocupar estos microhábitats (WEYGOLDT, 1969; ADIS et al., 1988; BELOZEROV, 2013).

Los fragmentos de bosque de manglar evaluados, se caracterizan por presentar una estructura vegetal heterogénea, el sector conocido como Playa Blanca registró los mayores valores de abundancia de adultos y ninfas para todas las especies, lo cual, 
sugiere una estrecha relación de la abundancia de los pseudoescorpiones con la estructura vegetal de la zona; es decir, el número de adultos (machos y hembras) y ninfas registrados para cada una de las especies, sugiere que los microhábitat donde se encuentran establecidos, ofrecen, todas las condiciones necesarias tanto de refugio como de alimento, tanto para adultos como para ninfas, así como de reproducción (WEYGOLDT, 1969; GABBUTT, 1970; MORÁIS y ADIS, 1997; AGUIAR et al., 2006). Sin embargo, los bajos valores de abundancia de ninfas registradas en las especies $L$. chthoniiformis, $P$. quirosi y Epactiochernes $\mathrm{sp}$., se asocia con el comportamiento críptico y edáfico de estas especies y, por lo tanto, dificultan el registro de los respectivos instar y la identificación de su periodo de reproducción (ADIS y MAHNERT, 1990; MORÁIS y ADIS, 1997; AGUIAR et al., 2006; AGUIAR y BÜHRNHEIM, 2011). Aunque, el descenso en el número de ninfas también se asocia con la mortalidad, al ser más susceptibles a los depredadores (WEYGOLDT, 1969; MORÁIS y ADIS, 1997; BELOZEROV, 2013).

La estructura-edad registrada y los ciclos de reproducción univoltino y bivoltino, sugieren una verdadera adaptación de estos arácnidos a los cambios producidos por el ciclo unimodal-biestacional dominante en la zona. La proporción sexual exhibida por la mayoría de las especies, parece indicar, que los recursos que ofrece el bosque de manglar son suficientes para obtener un éxito reproductivo, y que el dimorfismo sexual representado en el pequeño tamaño de los machos, sugiere que existe una competencia indirecta por tener acceso a las hembras. No obstante, la proporción sesgada hacia las hembras de Epactiochernes sp., parece indicar un comportamiento particular, en la que algunas especies de la familia, registran una fuerte competencia entre los machos por las hembras, generando implicaciones sobre la descendencia; sin embargo, la proporción sexual sesgada, exhibida necesita de un mayor estudio, debido a que, su hábito críptico hace difícil registrar eventos reproductivos.

\section{Agradecimientos}

Al Dr. Mark Harvey, Western Australian Museum, Australia; Dr. Volker Mahnert, Muséum d'histoire Naturelle de la Ville de Genève, Suiza; Dr. Giulio Gardini (Genova, Italia), por su asesoría y colaboración en el trabajo de identificación taxonómica, al Dr. Everton Tizo Pedroso, Universidad Federal de Goias (Goias, Brasil) por su colaboración, al Dr. Juan Zaragoza (Universidad de Alicante, España) por sus comentarios y asesorías, al personal de los Laboratorios de Entomología y de microscopia de la Universidad de Córdoba por su colaboración, finalmente, agradecemos a Juan Vergara y Maira Acosta Berrocal biólogos en formación de la Universidad de Córdoba por su ayuda en la colecta del material.

\section{Referencias}

ADIS, J.; JUNK, W. 2002. Terrestrial invertebrates inhabiting lowland river floodplains of Central Amazonia and Central Europe: a review. Freshwater Biology 47:711-731.

ADIS, J.; MAHNERT, V. 1985. On the natural history and ecology of pseudoscorpiones (Arachnida) from an Amazonian backwater inundation forest. Amazoniana 9 (3):297-314.

ADIS, J.; MAHNERT, V. 1990. Vertical distribution and abundance of pseudoscorpion species (Arachnida) in the soil of a neotropical secondary forest during the dry and the rainy season. Acta Zoological 190:11-16.

ADIS, J.; MAHNERT, V. 1993. Vertical distribution and Abundance of pseudoscorpions (Arachnida) in the soil of two different neotropical primary forests during the dry and rainy seasons. Mem of the Queens Museum 33 (2):431-440.

ADIS, J.; MAHNERT, V.; MORAIS, J.; RODRIGUES, J.1988. Adaptation of an Amazoniaian pseudoscorpion (Arachnida) from dryland forests to inundation forests. Ecology 69 (1):287-291.

AGUIAR, N.O.; BUHRNHEIM, P.F. 2003. Pseudoscorpiones (arachnida) Da Vegetaca De Sub-Bosque Da Floresta Primaria Tropical De Terra Firme, (Coari, Amazonas, Brasil). Acta Amazonica 33:515-526.

AGUIAR, N.O; BÜHRNHEIM, P.F. 2011. Pseudoscorpionida (Arachnida) em galerias de colônias de Passalidae (Coleoptera, Insecta) em troncos caídos em floresta de terra firme da Amazônia, Brasil. Acta amazónica 41 (2):311 - 320.

AGUIAR, N.O; GUALBERTO, T.; FRANKLIN, E. 2006. A medium-spatial scale distribution pattern of Pseudoscorpionida (Arachnida) in a gradient of topography (altitude and inclination), soil factors, and litter in a central Amazonia forest reserve, Brazil. Brazilian Journal of Biology 66:791-802. 
BELOZEROV, N.V. 2013. Seasonal aspects of the lifecycles of pseudoscorpions (Arachnida, Pseudoscorpiones). Entomological Reviews 93 (5):634-652.

BUDDLE, C. 2010. Photographic key to the Pseudoscorpions of Canada and the adjacent USA. Canadian Journal of Arthropod Identification 2002 10:1-77.

BUNNEFELD, N.; BAINES, D.; NEWBORN, D.; MILNER-GULLAND, E. 2009. Factors affecting unintentional harvesting selectivity in a monomorphic species. J Animal Ecol. 78:485-492.

CARVALHO, L.S.; DIAS, S.C; DAVID F. CANDIANI, D.F.; BONALDO, A.B. 2010. On the female of Metagonia taruma (Araneae: Pholcidae), ecology of the pholcid spiders in the Urucu River Basin, Amazonas, Brazil and new records from Brazilian Amazonia. Zoologia 27 (3):431-439.

CHAMBERLIN, J. 1931. The arachnid order Chelonethida. Stanford University Publications, Biological Sciences 7 (1):1-284.

CORTÉS, D.V.; RANGEL, J.O. 2011. Los bosques de mangle en un gradiente de salinidad en la Bahía de Cispatá - Boca Tinajones, departamento de Córdoba-Colombia. Caldasia 33 (1):155-176.

CVS-INVEMAR. 2010. Plan integral de manejo del distrito de Manejo Integrado (DMI) bahía de Cispatá - La Balsa - Tinajones y sectores aledaños del delta estuarino del río Sinú, departamento de Córdoba. Serie de Publicaciones Especiales No. 18 de INVEMAR. Santa Marta.

DEL-CLARO, K.; TIZO-PEDROSO, E. 2009. Ecological and evolutionary pathways of social behavior in Pseudoscorpions (Arachnida: Pseudoscorpiones). Acta Ethological 12:13-22.

FISHER, R. 1930. Genetical Theory of Sex Allocation. Claren don Press, Londres.

FOELIX, R. 2011. Biology of spiders. 3 ed. Oxford Univ Press, Oxford.

GABBUTT, P.D. 1962. Nets of the marine falsescorpion. Nature London 196:97-98.

GABBUTT, P.D. 1966. An Investigation of the Silken Chambers of the Marine Pseudoscorpions Neobisium maritimun. Jorunal of Zoology Londond 149:337-343.

GABBUTT, P.D. 1970. Samplign Problems and the validity of the life history analyses of pseudoscorpions. Journal of Natural History 4:1-15.

GABBUTT, P.D.; VACHON, M. 1965. The external morphology and life history of the pseudoscorpion Neobisium muscorum. Proceedings of the Zoological Society of London 145:335-358.

GRETHER, G.F.; ALLER, T.L.; GRUCKY, N.K.; LEVI, A.; ANTAKY, C.C.; TOWNSEND, JR. V.R. 2014. Species differences and geographic variation in the communal roosting behavior of Prionostemma harvestmen in Central American rainforests. The Journal of Arachnology 42:257-267.

HAMILTON, W.D. 1967. Extraordinary sex ratios. Science 156:477-488.

HARVEY, M. S. 2009. The first Australasian species of the genus pseudoscorpion halophilic Paraliochthonius (Pseudoscorpiones: Chthoniidae). Records of the Western Australian Museum 25:329-344.

HARVEY, M. S. 2013. Pseudoscorpions of the World, version 2.0. Western Museum Australian. Disponible en: URL: Http:www.museum.wa.gov.au /catalogs/pseudoscorpion. Consultado: 5-12-2015.

HJERNQUIST, M.B.; HJERNQUIST, K.A.T.; FORSMAN, J.T.; GUSTAFSSONA, L. 2009. Sex allocation in response to local resource competition over breeding territories. Behavioral Ecology 20:335-339.

HOFF, C. 1959. The ecology and distribution of the pseudoscorpions of north-central New Mexico, University of New Mexico Publications in Biolgy 8:1-68. 
JUDSON, M. 1992. A simple, Slow-diffusion Method for clearing small arthropods. Newsl Br. Arachnology Society 64:6-7.

JUDSON, M. 1994. Les Pseudoscorpions. Pénelope 12:6-37.

KASSIRI, H.; KASIRI, N.; DIANAT, A. 2015. Species composition, sex ratio, geographical distribution, seasonal and monthly activity of scorpions and epidemiological features of scorpionism in Zarrin-dasht County, Fars Province, Southern Iran. Asian Pacific Journal of Tropical Disease 5 (1):S99-S103.

KENSLER, C. 1967. Dessication resistance of intertidal crevice species as a factor in their zonation. Journal of Animal Ecology 36:391-406.

LEE, V. 1979. The maritime pseudoscorpions of Baja California, México (Arachnida: Pseudoscorpionida). Occasional Paper of the California Academy of Sciences 131 (i-iv):1-38.

LEGG, G. 1974. An account of the genital musculature of pseudoscorpions (Arachnida). Bulletin British Arachnology Society 3:38-41.

LEGG, G. 1975. Ageneralised account of the male genitalia and associated glands of pseudoscorpions (Arachnida). Bulletin of the British Arachnological Society 3:66-74.

MACHADO, E.O.; BRESCOVIT A.D.; CANDIANI, D.F.; HUBER, B.A. 2007. Three new species of Mesabolivar (Aranea, Pholcidae) from leaf litter in urban environments in the city of São Paulo, São Paulo, Brazil. Iheringia, Sér. Zool., Porto Alegre 97 (2):168-176.

MAHNERT V. 2014. Pseudoscorpions (Arachnida: Pseudoscorpiones) from the Galapagos Islands (Ecuador), Revue Suisse de Zoologie 121 (2):135-210.

MAHNERT, V.; ADIS, J. 2002. Pseudoscorpiones. Págs. 367-380. En: Adis, J. (ed). Amazonian Arachnida and Myriapoda. Pensoft Publisher. Sofía, Moscow.

MAHNERT, V.; SCHUSTER, R. 1981. Pachyolpium atlanticumn. sp., ein Pseudoskorpion aus der Gezeitenzone der Bermudas - Morphologie und Ökologie (Pseudoscorpiones: Olpiidae). Rev Suis Zool. 88:265-273.

MERINO-SÁINZ, I.; FERNÁNDEZ-ÁLVAREZ, F.A.; PRIETO, C.E. 2013. Nuevos datos sobre Megabunus diadema (Fabricius, 1779) (Opiliones: Phalangiidae). Revista Ibérica de Aracnología 22:102-106.

MORÁAIS, J.; ADIS, J. 1997. Abundance and phenology of Pseudoscorpiones (Arachnida) from a mixedwater inundation forest in Central Amazonia, Brazil. Revue Suisse de Zoologie 104:475-483.

OMOLOYE, A. 2006. Sex ratio bias in the F1 adult progeny of African rice gall midge, Orseolia oryzivora H, and G. (Dipt, Cecidomyiidae). Journnal of Applied Entomology 130:349-355.

POLIS, G.A. 1990. The Biology of Scorpions. Ed. Stanford University Press, Stanford, California.

RANIUS, T.; DOUWES, P. 2002. Genetic structure of two pseudoscorpion species living in tree hollows in Sweden. Animal Biodiversyt and Conservation 25 (2):67-74.

ROJAS, P. 1989. Entomofauna asociada a los detritos de Atta mexicana (F. Smith) (Hymenoptera: Formicidae) en una Zona Árida del Centro de México. Acta Zoológica Mexicana 33:1-51.

SCHUSTER, R. 1962. Das marine Litoral als Lebensraum terrestrischer Kleinarthropoden. Internatione Revue der Gesamten Hydrobiologie 47:359-412.

SMITH, R. 1999. Statistics of sexual size dimorphism. Journal of Human Evolution 36:423-459.

TABADKANI, S.; ASHOURI, A.; RAHIMI-ALANGI, V.; FATHI-MOGHADDAM, M. 2012. When to estimate sex ratio in natural populations of insects? A study on sex ratio variations of gall midges within a generation. Entomologica Sciences 2:1-6. 
TIZO-PEDROSO, E.; DEL-CLARO, K. 2007. Cooperation in the neotropical pseudoscorpion, Paratemnoides nidificator (Balzan, 1888): feeding and dispersal behavior. Insectes Sociaux 54:124-131.

TRYJANOWSKI, P.; SPARKS, T.; KAMIENIARZ, R.; PANEK, M. 2009. The relationship between hunting methods and sex, age and body weight in a non-trophy animal, the red fox. Wild Rese 36:106-109.

VANIN, S.; TURCHETTO, M. 2007. Winter activity of spiders and pseudoscorpions in the South-Eastern Alps (Italy). Italian Journal of Zoology 74 (1):31-38.

VOLLRATH, F. 1986. Eusociality and Extraordinary Sex Ratios in the Spider Anelosimus eximius (Araneae: Theridiidae). Behavioral Ecology and Sociobiology, 18 (4):283-287.

WADE, M.J.; SHUSTER, S.M.; DEMUTH, J.P. 2003. Sexual Selection Favors Female-Biased Sex Ratios: The Balance between the Opposing Forces of Sex-Ratio Selection and Sexual Selection. The American Naturalist 162 (4):403-414.

WEDEKIND, C. 2012. Managing Population Sex Ratios in Conservation Practice: How and Why? Págs. 81-96 en: Povilitis, T. (ed.), Topics in Conservation Biology: Rijeka and Shangai. InTech press.

WEYGOLDT, P. 1969. The biology of pseudoscorpions. Harvard Books in Biology. 6 ed. German edition. Cambridge, Massachusetts.

YSNEL, F. 1991. Data points for a study of population dynamics of an Orb-Weaving spider (Larinioides cornutus, Araneae, Araneidae). Bulletin Scoiety neuchatel Science Nature 116 (1):269-278.

ZEH D.W. 1987a. Life history consequences of sexual dimorphism in a chernetid pseudoscorpion. Ecology 68:14951501.

ZEH, D.W. 1987b. Aggression, density, and sexual dimorphism in chernetid pseudoscorpions (Arachnida: Pseudoscorpionida). Evolution 41:1072-1087.

ZEH, D.W.; ZEH, J.A. 1997. Sex via the substrate: mating systems, sexual selection and speciation in pseudoscorpions. Págs.329-339 en: Choe, J.C.; Crespi, B.J. (ed.), The evolution of mating systems in insects and arachnids. Cambridge, Massachusetts.

ZEH, J.A.; ZEH, D.W. 1990. Cooperative foraging for large prey by Paratemnus elongatus (Pseudoscorpionida, Atemnidae). Journal of Arachnology 18:307-311.

ZEH, J.A.; ZEH, D.W. 1992. Are sexually-selected traits reliable species characters? Implications of intra-brood variability in Semeiochernes armiger (Balzan) (Pseudoscorpionida: Chernetidae). Bulletin of the British Arachnological Society 9:61-64. 